\title{
Recurrent craniopharyngioma with extensive necrosis and epithelial reactive changes: Are we facing apoplexy driven by premalignant changes? Two case reports
}

\author{
Martha Lilia Tena-Suck ${ }^{1 *}$, Alma Ortiz-Plata ${ }^{2}$, Laura Chavez Macias ${ }^{3}$, Erick Gomez Apo ${ }^{3}$, Carlos Peñafiel-Salgado ${ }^{1}$ and Citlaltepetl Salinas-Lara ${ }^{1}$
}

*Correspondence: mltenasuck@gmail.com

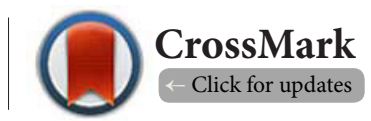

'Department of Neuropathology, National Institute of Neurology and Neurosurgery, Mexico City.
'Laboratory of Experimental Neuropathology. National Institute of Neurology and Neurosurgery, Mexico City.
${ }^{3}$ Department of pathology. General Hospital of Mexico City.

\begin{abstract}
Pituitary apoplexy is characterized by a sudden increase in pituitary gland volume secondary to ischemia and/or necrosis, usually occurring in the context of pituitary adenoma. Little is known about craniopharyngioma apoplexy. However, some cases have been reported, and it is usually an underused but necessary term in the presence of extensive necrosis and hemorrhage within Craniopharyngiomas. Here, we report two cases of craniopharyngioma apoplexy in a man aged 26 and a woman aged 30 years old, who presented this sellar tumor at an early age, both presenting recurrence and had previously undergone radiotherapy. In the previous recurrence, we observed histological findings related to extensive necrosis, fibrin thrombi, vascular damage and proliferation, inflammation, hemorrhage, fibrin foci, and epithelial reactive changes. Also, we observed pseudostratification, atypia and external epithelium pleomorphism, proliferation of the stellate reticulum with mitotic features as well. Strikingly, by immunohistochemistry, we described the overexpression of $\beta$-catenin only in loose cells found the middle of the extensive necrosis areas, displaying a high Ki67 index, found in its punctate form. Both cases showed the same histological pattern. Such findings hadn't been described in Craniopharyngiomas; we suggest that these are related to apoplexy, post radiation changes or premalignant transformation, reason why this could be termed carcinoma in situ or high-grade intraepithelial lesion. The 2016 WHO classification for CNS tumors update does not consider new features related to malignant transformation occurring in Craniopharyngiomas.
\end{abstract}

Keywords: Craniopharyngioma, apoplex, necrosis, malignant transformation, ki67

\section{Introduction}

The term "pituitary apoplexy" (PA) is an uncommon clinical condition in which a pituitary tumor (PTs) outgrows its blood supply and a stroke overcomes [1], sometimes with considerable, abrupt hemorrhage, leading to a sudden onset of pituitary necrosis and compressive symptoms. PA is considered a neurosurgical emergency. The incidence of PA has been described in up to $10.11 \%$ of patients with pituitary macroadenoma (PMA), and in $0.36 \%$ of patients with microadenomas [2]. Precipitating factors include increase in intracranial pressure, arterial hypertension, major surgery, anticoagulant therapy, dynamic testing, high altitude, stress, or pregnancy [2]. While PA occurs mostly in adenomas; it has also been described in non-adenomatous sellar lesions including hypophysitis, sellar metastases, especially from Rathke's cleft cyst, sellar tuberculoma and craniopharyngioma [2].

Craniopharyngiomas (CPs) account for $2.5 \%$ of all intracranial neoplasms [3]. Regardless of their typical localization in the suprasellar region and adjoining structures, $10-20 \%$ of them occupy intrasellar area, but only very few grow as intrasellar cysts [3]. Symptomatic hemorrhage in cystic craniopharyngioma may mimic pituitary apoplexy; nonetheless, etiology is relatively different. Necrosis, hemorrhage and degenerative changes are neither common nor characteristic in CPs, and are usually associated with malignant transformation (MT) [3].

The aim of this paper is to present two unusual cases of

(c) 2020 Tena-Suck et al; licensee Herbert Publications Ltd. This is an Open Access article distributed under the terms of Creative Commons Attribution License (http://creativecommons.org/licenses/by/3.0). This permits unrestricted use, distribution, and reproduction in any medium, provided the original work is properly cited. 
aggressive craniopharyngioma that showed necrosis and hemorrhage with reactive epithelial changes that might suggest premalignant histological changes or apoplexy as a differential.

\section{Clinical cases presentation \\ Case 1}

A 30-year-old woman presents to the neurological emergency room with epistaxis. She had a history going twelve years back, when at 18 she developed persistent headache, nausea and visual disturbances (right temporal hemianopia). MRI showed a parasellar lesion, so surgical approach was performed. Tumor was removed and histopathological examination revealed an adamantinomatous craniopharyngioma, which corresponds to grade I in the WHO classification system [3]. Subsequently, she undergone radiation therapy and remained asymptomatic for 6 years, though for the following six years she'd be operated six times due to tumor recurrence. She underwent a trans sphenoidal biopsy corresponded to AdaCP by biopsy and last MRI showed extensive tumor infiltrating skull base (Figure 1a). Surgical team performed partial resection of the lesion and intra-operative study was requested, which was reported as a malignant sellar tumor. Cytopathological analysis revealed epithelial cells with prominent nucleoli and some cellular pleomorphism with necrotic background (Figures $\mathbf{1 b}$ and $1 \mathrm{c}$ ).

\section{Case 2}

A 26-year-old man diagnosed of craniopharyngioma eleven

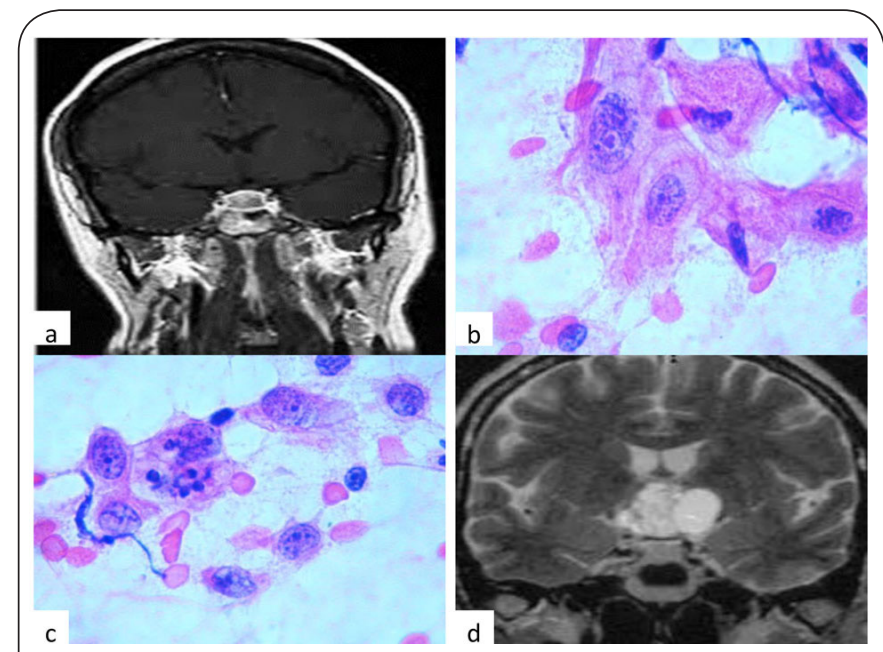

Figure 1. (a) MRI Imaging reveals a partly cystic, partly contrast enhancing intra--/suprasellar mass lesion. (b) Intraoperative analysis of the case 1, shows some cellular smears with atypia, in (c) observed epithelial cells with nucleoli and necrosis background (H\&Ex 400). MRI reveals a heterogeneous parasellar tumor of the case two in (d). Histological findings show a tumor with extensive coagulative necrosis in (e), hemorrhage in (f), with thrombus and fibrin deposition in (g), and in (h) observe macrophages and granuloma for mation (H\&Ex 400). years prior to the current moment, having had his first surgery resection was at 15 . He was treated with radiotherapy and stayed asymptomatic for 6 years, at 21 he was re-operated, and craniopharyngioma diagnosis kept unchanged. He received radiotherapy again and remained asymptomatic for 3 years. Now, he presented to the emergency room reporting rapidly progressive visual acuity decrease until bilateral amaurosis, intense headache and clinical manifestations of intracranial hypertension. MRI showed a heterogeneous tumor portraying extensive infiltration to the skull base (Figures $1 \mathrm{~d}$ and $1 \mathrm{e}$ ), and he underwent surgery again. Histological image changed in respect to the two previous surgeries showing a tumor with extensive necrosis and hemorrhage.

\section{Histopathology}

Both cases were initially diagnosed as adamantinomatous $C P$, and both showed the same histological pattern in the most recent biopsy, which drew our attention due to two particularly characteristic patterns. On the first place, most of the tumor showed coagulative necrosis (Figures $\mathbf{2 a}, \mathbf{2 b}, \mathbf{2 c}$ and $\mathbf{2 d}$ ), thrombi and fibrin deposition (Figures $2 \mathrm{e}$ and $2 \mathrm{f}$ ), an acute, dense inflammatory infiltrate (Figure $\mathbf{2 g}$ ), macrophages and granuloma formation (Figure $\mathbf{2 h}$ ). On the other hand, a second pattern, localized in the external epithelium, showed atypical cellularity (Figures $3 \mathbf{3 a}$ ), pseudostratification (Figure $\mathbf{3 b}$ ) with spindle cells (Figure $3 \mathrm{c}$ ), scarce mitotic figures (Figures $\mathbf{3 d}$ and $3 \mathbf{3 e}$ ), loss of polarity (Figure 3f), and hypercellular stellate reticulum with basaloid appearance (Figure $3 \mathrm{~g}$ ), necrosis and mitotic figures in the stellate reticulum (Figure $3 \mathbf{h}$ ). Immunohistochemically analysis was performed and showed that the external epithelium was strongly positive for keratin $7 / 8 / 18$ (Figure 4a), small cell nests in the middle of necrotic areas also showed also $\square$-catenin positivity (Figure $4 \mathbf{4 b}$ ). Vimentin was expressed the stellate reticulum cells and the outer edge of the epithelium (Figure 4c), furthermore, the abundance of blood vessels is striking (Figure 4d), along with fibroblast prolifera-

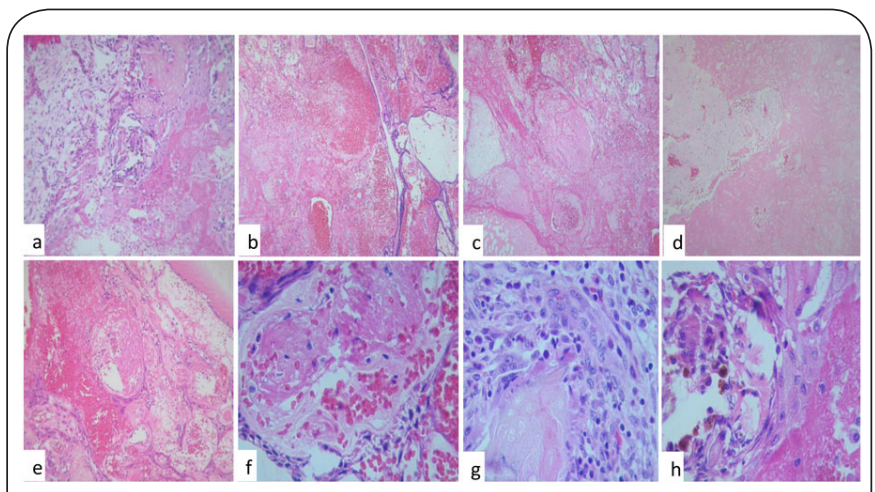

Figure 2. Histopathology on the first place, most of the tumor showed coagulative necrosis (a), (b), (c) and (d), thrombi and fibrin deposition in (e), and (f), an acute, dense inflammatory infiltrate (g), macrophages and granuloma formation in (h) (original magnification H\&E x 400). 
tion (Figure 4d). This region showed avid positivity for EVFG (Figure 4e), CD34, Factor VIII, EGFR, EGF, BFGF, PDGF (Figure 4f). Immunoexpression of p53 and p63 was more intense in the areas of pseudostratified epithelium. Ki67 index was also positive in nuclei, with a punctate appearance (Figure $\mathbf{4 f}$ and $\mathbf{4 g}$ ). Based on the histopathological findings, adamantinomatous craniopharyngioma with extensive necrosis (apoplexy) and reactive changes were diagnosed in both cases.

\section{Discussion}

An infrequent complication, sellar region apoplexy has been described in some rare presented cases associated to Rathke's cleft cyst and pituitary adenomas, but this event has not been

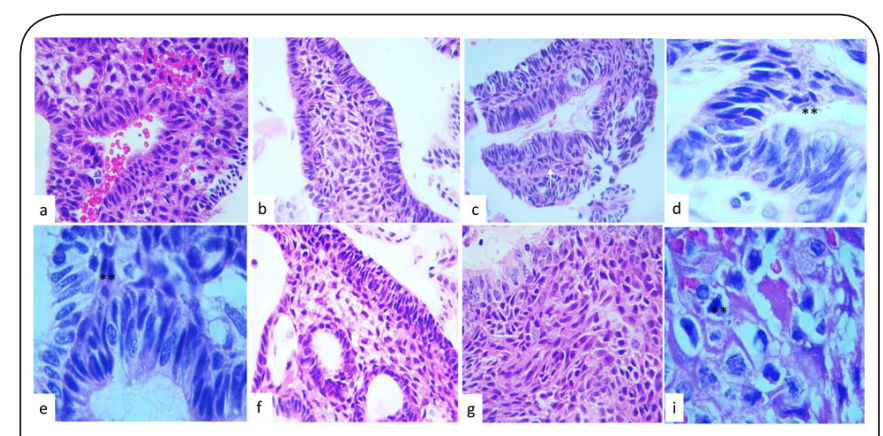

Figure 3. The histopathological appearance of the second pattern is related to the outer epithelium that it presents atypical cellularity (a), pseudo stratification of the external epithelium in (b), with spindle cells in (c), (d). And in (e) there are scarce mitotic figures in the external epithelium. (f) Shows loss of polarity of the external epithelium (f) and hypercellular stellate reticulum with basaloid appearance in (g) and necrosis and mitosis features in RS were observed (h). Asterisks show the mitotic figures (H\&Ex400).

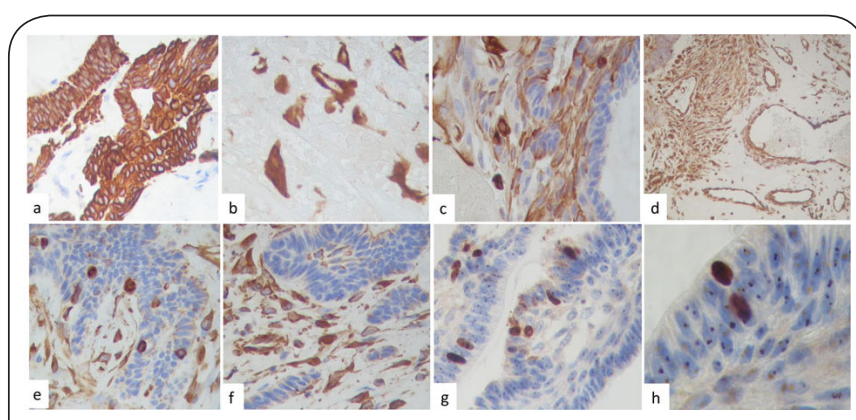

Figure 4. Immunohistochemistry.

(a) Keratin 7 positive immunoreactions in the outer epithelium, (b) show the $\beta$ catenin immunoreaction in small nest keratin positive in necrosis areas (b) Vimentin was positive in cells of the stellate reticulum and the outer edge of the epithelium (c), and in (d) numerous vessels and fibroblast proliferation were observed in (d). Strongly positive immunoreaction for EVFG (e), and PDGF (f) in vessels and fibroblast proliferation are seen Ki-67 (g) shows more positivity reaction from normal appearance and in (h) is displayed Ki-67 positive reaction in the nucleoli and with a punctate appearance (IHQ stain x 400 deeply analyzed in the context of craniopharyngioma. Clinical manifestations are similar to those found in pituitary tumor apoplexy. Acute headache is the most dominant presentation. Out patients' severe, sudden onset headaches, along with their individual history of CP was highly suggestive of apoplexy, causing intracranial hypertension syndrome, believed to result from the sudden expansion of the mass in the sella turcica, also, there was strong radiologic evidence of hemorrhage and acute hydrocephalus. MRI also indicated large cystic lesions causing mass effect, which was also proven by intraoperative findings before excision. Furthermore, both patients complained of acute visual acuity deterioration.

PA is characterized by acute ischemic and/or necrotic infarction, in pituitary adenoma [1,2]. Apoplexy can occur in Rathke's cleft cyst as well as in CP. Clinical manifestations of the patients with RCC apoplexy are similar to those with pituitary tumor apoplexy [2]. Brain imaging is required to identify a sellar lesion which confirms the diagnosis by revealing a heterogeneous, sometimes calcified and cystic tumor with hyperintense (hemorrhagic) and/or hypo intense (necrotic) components [2]. Detailed pathophysiology is not completely understood. However, widely accepted hypotheses consider tumor vascular occlusion due to tumor rapid overgrowth, tumor blood flux reduction and tumoral aberrant vascularization. Other theories postulate acute rise in blood flow due to systemic hypertension or physical activity, reduction of vascular flux caused by surgery, radiotherapy or post intrathecal anesthesia. Molecular evidence shows abnormal expression of VEGF mRNA, coagulation disturbances such as thrombocytopenia or pharmacological anticoagulation, pregnancy, diabetes mellitus, estrogen replacement therapy, dopamine agonist therapy, sickle cell anemia, lymphocytic leukemia and head trauma in an another hang [2,6]. Differential diagnoses include meningitis, subarachnoid hemorrhage, midbrain infarction, cavernous sinus thrombosis, hemorrhagic infarction in a Rathke's cleft cyst and aneurysms [2]. Hemorrhage and necrosis are not common histological findings in CP but the etiology seems to be quite different. Minor hemorrhage may recur unless the cyst wall is totally removed. When CP walls break, the exit of the cholesterol-rich OMF can cause angiogenic, hypoxic effects, vasoconstriction and chemical meningitis, inducing vasospasm [8], inflammatory or thromboembolic complication [9]. Furthermore, radiation-induced vascular abnormalities are considered strong risk factors to malignant transformation and stroke. Early postoperative complications of CPs include evidence of hypothalamic or pituitary iatrogenic injury, hormonal insufficiency, metabolic changes, of seeding [6].

How infrequent is it for CPs, anyway, to undergo malignant transformation? The previous report considered malignant appearance as tumor cells showed moderate to severe pleomorphism, hyperchromatic nuclei, increased nuclear: cytoplasmic ratio, high mitotic activity, focal coagulative necrosis, basement membrane destruction, infiltrative growth 
and microvascular proliferation. The malignant phenotypes that have been reported in $\mathrm{CP}$ are the following: squamous cell carcinoma, odontogenic ghost cell carcinoma, low-grade myoepithelial carcinoma, one case amelomeloblastic carcinoma and basaloid type carcinoma. In situ carcinoma is a rare event described in CPs. The mechanisms for CP malignant transformation are still unknown. So far, these are the first reported couple of craniopharyngioma apoplexy cases with the association of radiotherapy-induced malignant transformation, and it adopts a wide arrange of histological appearances [10]. Time intervals of transformation from primary tumor to malignancy ranged from 1.2 to 35 years (average 9.7 years) in the different cases reported [10]. However, it is necessary to consider and to be prudent when differentiating radionecrosis from tumor recurrence, which represents a major issue in neurooncology.

In our cases we observed necrosis and hyalinized, glomeruloid appearance of the wall of the vessels, which has also been reported elsewhere [7]. Definitive diagnostic criteria for malignant craniopharyngioma have not been established. In this case, we could consider that the radionecrosis does not persist for more than 10 years and necrosis observed in our patient is a recent event. In these cases, not only did we see necrosis, but also abundant vessels, fibroblast proliferation, epithelial cell detritus, wet keratin, many ghost cells in the areas of necrosis and epithelial changes in cystic structures. Although these changes have not been described as premalignant changes or perhaps consider calling them as a low-grade intraepithelial lesion or just call them secondary to necrosis reactive changes. Noteworthy, we observed an increase of Ki-67, p53, and p63 immunostaining in the pseudostratified epithelium areas. This punctate pattern of Ki67 represents an interesting finding that had not been notified in Craniopharyngiomas, and its positivity indicates nucleolar activity in these same areas, which may be indicative of incipient malignant changes, and they do not correspond to necrotic nor apoptotic changes. Furthermore, transoperative cytopathological analysis showed prominent nucleoli and atypical cellular changes were appreciated and, as mentioned, these are unusual in CP. Such findings may arise whether from the mere disease mechanisms or from the effects of treatment. Intracystic bleomycin (ICB) administration represents an effective treatment strategy for cystic craniopharyngioma and rarely causes serious complications. The authors report a case of vasculopathy after ICB injection for a recurrent cystic CRP. Conventional factors that increase the risks of cardio- and cerebrovascular diseases and DM and risks for developing new intracranial tumors contributed to excess morbidity and mortality. Comorbidities such as diabetes and cardiovascular and cerebrovascular disease, as well as risks for developing new intracranial tumors contributed to excess morbidity and mortality. These studies have identified increased risk of cerebrovascular disease, including stroke and Moya-Moya syndrome. A thromboembolic origin is suggested to originate this unusual event, due to the evidence shown by studies showing inflammatory vascular involvement after an episode of aseptic meningitis.

Aberrant vascular development was described as an increase in the number and density of abnormal vessels characterized by fibroblastic proliferation and hyalinization of the wall, extensive necrosis, thrombosis and epithelial changes. Thus, we posed ourselves the question whether these findings were caused by post radiation therapy changes, radionecrosis, or if it corresponded to a spontaneous necrosis secondary to malignant transformation, or whether it corresponds to a stroke, in a persistent tumor. The aberrant membranous $\beta$-catenin expression has been correlated to poor survival in patients with craniopharyngioma, as an independent risk factor in predicting the prognosis of this disease [12].

According to the 2016 WHO classification of CNS tumors update, there are no changes related to the malignant transformation of the CPs and maybe it would be like nonendocrine sellar tumors and as other tumors arising in the sellar region [13].

\section{Conclusion}

Craniopharyngioma is rarely associated to intratumoral hemorrhage and extended necrosis. CP apoplexy is a vascular condition which may be consequence of the interaction between high relapse incidence and radiation therapy. Here, we reported rare and overlapped morphological, clinical and immunohistochemical features of highly recurrent $\mathrm{CP}$, such synergistic coexistence created a clinical condition difficult to diagnose and manage. We are unaware whether the extensive necrosis and intraepithelial reactive changes are enough evidence to postulate that molecular findings and histological patterns are those of low grade intraepithelial lesion, though its highly aggressive behavior, along with its particularly elevated recurrence rate, may support its inclusion into the definition of malignancy. We hope that our detailed cases will prove to be helpful in further founding the diagnostic criteria and evaluation of biological behavior and pathophysiological mechanisms for malignant transformation.

\section{List of abbreviations}

PA: Pituitary Apoplexy

PT: Pituitary Tumor

PA: Pituitary Adenomas

PMA: Pituitary Macroadenomas

CPs: Craniopharyngiomas

MRI: Magnetic Resonance Imaging

OMF: Oil Machinery Fluid

DC: Dystrophic Calcifications

EVGF: Endothelial Vascular Growth Factor

EGF: Epithelial Growth Factor

BFGF: Beta Fibroblast Growth Factor

PDGF: Platelet Derived Growth Factor

WHO: World Health Organization 
Tena-Suck et al., Journal of Histology \& Histopathology 2020, http://www.hoajonline.com/journals/pdf/2055-091X-7-2.pdf

\section{Competing interests}

The authors declare that they have no competing interests.

Authors' contributions

\begin{tabular}{|l|c|c|c|c|c|}
\hline Authors' contributions & MLTS & AOP & LCM & CPS & CSL \\
\hline Research concept and design & $\checkmark$ & -- & -- & -- & -- \\
\hline Collection and/or assembly of data & -- & $\checkmark$ & -- & -- & -- \\
\hline Data analysis and interpretation & $\checkmark$ & -- & -- & -- & -- \\
\hline Writing the article & $\checkmark$ & -- & -- & -- & -- \\
\hline Critical revision of the article & $\checkmark$ & -- & -- & -- & -- \\
\hline Final approval of article & $\checkmark$ & -- & -- & -- & -- \\
\hline Statistical analysis & $\checkmark$ & -- & -- & -- & -- \\
\hline
\end{tabular}

Publication history

Editor: Khin Thway, The Royal Marsden Hospital, UK.

Received: 05-Dec-2019 Final Revised: 07-Jan-20

Accepted: 10-Feb-2020 Published: 20-Feb-2020
12. Li Z, Xu J, Huang S and You C. Aberrant membranous expression of betacatenin predicts poor prognosis in patients with craniopharyngioma. Ann Diagn Pathol. 2015; 19:403-8. | Article | PubMed

13. Lopes MBS. The $\mathbf{2 0 1 7}$ World Health Organization classification of tumors of the pituitary gland: a summary. Acta Neuropathol. 2017; 134:521535. | Article I PubMed

\section{Citation:}

Tena-Suck ML, Ortiz-Plata A, Macias LC, Apo EG, Peñafiel-Salgado C and Salinas-Lara C. Recurrent craniopharyngioma with extensive necrosis and epithelial reactive changes: Are we facing apoplexy driven by premalignant changes? Two case reports. J Histol Histopathol. 2020; 7:2. http://dx.doi.org/10.7243/2055-091X-7-2

\section{References}

1. Briet C, Salenave S and Chanson P. Pituitary apoplexy. Endocrinol Metab Clin North Am. 2015; 44:199-209. I Article I PubMed

2. Glezer A and Bronstein MD. Pituitary apoplexy: pathophysiology, diagnosis and management. Arch Endocrinol Metab. 2015; 59:259-64. | Article I PubMed

3. Rushing EJ, Giangiaspero F, Paulus W and Burger PC. Craniopharyngioma. In: World Health Organization classification of tumours: pathology and genetics of tumour of the Nervous System, ed. Louis DN, Ohgaki H, Wiestler OD, Cavenee WK 2007; IARC Press, Lyon, France. 238-240.

4. Rangel-Castilla L, Rios-Alanis M, Torres-Corzo J, Rodriguez-Della Vechia R and Chavez-Lopez R. [Pituitary apoplexy as the presenting symptom of a recurrent craniopharyngioma]. Rev Neurol. 2004; 39:297-8. | Article | PubMed

5. Shida N, Nakasato N, Mizoi K, Kanaki M and Yoshimoto T. Symptomatic vessel narrowing caused by spontaneous rupture of craniopharyngioma cyst--case report. Neurol Med Chir (Tokyo). 1998; 38:666-8. | Article | PubMed

6. Ricarte IF, Funchal BF, Miranda Alves MA, Gomes DL, Valiente RA, Carvalho FA and Silva GS. Symptomatic Cerebral Vasospasm and Delayed Cerebral Ischemia Following Transsphenoidal Resection of a Craniopharyngioma. J Stroke Cerebrovasc Dis. 2015; 24:e271-3. | Article I PubMed

7. Wang W, Chen XD, Bai HM, Liao QL, Dai XJ, Peng DY and Cao HX Malignant transformation of craniopharyngioma with detailed followup. Neuropathology. 2015; 35:50-5. | Article | PubMed

8. Shida N, Nakasato N, Mizoi K, Kanaki M and Yoshimoto T. Symptomatic vessel narrowing caused by spontaneous rupture of craniopharyngioma cyst--case report. Neurol Med Chir (Tokyo). 1998; 38:666-8. | Article | PubMed

9. Fischer B, Palkovic S, Schildheuer D, Heindel W and Wassmann H. Inflammatory thromboembolic complication after craniopharyngioma surgery? Acta Neurochir (Wien). 2004; 146:183-6. | Article I PubMed

10. Wang W, Chen XD, Bai HM, Liao QL, Dai XJ, Peng DY and Cao HX. Malignant transformation of craniopharyngioma with detailed followup. Neuropathology. 2015; 35:50-5. | Article | PubMed

11. Wijnen M, Olsson DS, van den Heuvel-Eibrink MM, Hammarstrand C, Janssen J, van der Lely AJ, Johannsson G and Neggers S. Excess morbidity and mortality in patients with craniopharyngioma: a hospital-based retrospective cohort study. Eur J Endocrinol. 2018; 178:93-102. | Article | PubMed 